\title{
The Aesthetic Impression Given By Web-Based Learning Systems: The Influence of Cognitive Style
}

\section{Pei Shan Teng, National Yunlin University of Science and Technology, Taiwan \\ Dengchuan Cai, National Yunlin University of Science and Technology, Taiwan}

\begin{abstract}
Drawing on theories of cognitive psychology, I have developed a research model to explain the relationship between the visual factors and the aesthetic impression of a web page according to users' emotional responses. A series of investigations of a web-based learning system were explored in order to further understand the aesthetic impression created by different cognitive styles. The results indicated that: 1) 15 adjective pairs were classified two major visual factors, order and complexity, through semantic difference method and factor analysis; 2) a perceptual map of web-based learning systems was drawn up according to the two dimensions of complexity vs. simple, and order vs. confused; 3 ) the intermediate students showed higher emotional responses than holistic and analytic students especially in regard to the order factor when viewing a webpage.
\end{abstract}

Keywords: cognitive style, visual complexity and visual order, Web-Based learning system 


\section{Introduction}

Recent studies show that a greater number of people now learn skills, obtain and share information via websites or forums on the Internet. However, web users' browsing of web pages tends to depend not only on cognitive usability but also on emotional feelings. The quality of the studying environment has been overloaded with excessive information and chaotic visual design on the web pages. As users are presented with different websites on the Internet, they can switch effortlessly. Bucy (2000) indicated that people's choice of website may reflect their emotional feelings, i.e. whether they feel pleasure or enjoyment, when they gain their first impression of a website.

The influences of user interface design have received increasing attention (Dillon, 2001; Norman, 2002). A human being's affective system is judgmental, assigning a person's positive or negative dimension to the environment (Norman, 2002). Emotional responses emphasize the subjective nature of the user's experience of the attraction of the website'. It is closely linked to individual feeling, cognitions, and motivations. Cognitive style plays an important role in the viewing of web content because a web design should ultimately accommodate an individual's typical mode of perception, thinking, remembering, and problem-solving in order to promote usability. Therefore, good interface designs can enhance the user's capacity to process the information on the webpage.

\section{Individual Differences: Cognitive Style}

The term "cognitive style" has been used in the field of individual differences to describe a set of qualities, activities and behaviors that every person exhibits in a persistent way. In education, the concepts of cognitive style have been explored extensively (Riding, 1998) and, 
although these have been used interchangeably, cognitive style is the "individual's preferred and habitual approach to organizing and representing information" (Riding, 1998). Riding and Cheema (1991) pointed out differences between those who organize information as wholes, who are labeled 'wholists' and those who break down information into smaller parts who are labeled 'analytics'. Therefore, the development of personal abilities and specialties can enhance learning interest and lead to greater achievement through an appropriate learning environment from an educational viewpoint.

\section{Visual Complexity and Visual Order}

Thompson and Robitaille (1992) argued that the aesthetic impression is an important factor in evoking user pleasure and preference. When the aesthetic appearance of a website is more attractive the user's emotional response to it is greater. Moreover, a few studies have attested to the significant effects of visual complexity and visual order on web design, web qualities and visual preference (Deng and Poole, 2010; Arnheim, 1966; Lavie, 2004). Web page visual complexity and order are not clearly defined in Information System research. Therefore, Arnheim's concepts of environmental complexity and order may be extended to the website context or emotional responses.

Recently research has been conducted on applying aesthetics to interface designs. However, in contrast with the ample amount of research focusing on the cognitive usability of interfaces (Cockton, 2002), only a few studies have focused on the aesthetic aspects of a web page. Tsai and Chang (2008) indicated that the degree of visual complexity was closely related to emotional responses and usability.

\section{Measure of Emotion}


The emotional space can be constructed in terms of the major aesthetic dimensions, which can be measured by the semantic differential method (SD) (Osgood, 1957). The SD method has been used successfully as the basis for applying Kansei (Sensational) engineering, Kansei being a set of methods used for designing the aesthetic aspects of physical products, such as automobiles, office chair design (Jindo etc, 1995). Using SD methods, each aesthetic dimension is defined by a set of pairs of polar adjectives, such as 'Beautiful-ugly, warmcold' and is assumed to represent a straight-line function that passes through the origin of the emotional space (Tsai and Chang, 2008; Chuanga and Ma, 2001).

Recently, the multidimensional scaling method (MDS), a method for recovering the perceptual space of stimuli from the subjects' judgments of similarity among stimuli, has been applied to investigate users' perception and preference of product types (Green and Carmone, 1989; Schenkman and Jönsson, 2000). Hence, MDS makes it possible for researchers to understand underlying psychological structures of stimuli, and not only surface behavior, which is visible to an observer.

The aesthetic impressions of web pages depend upon many interrelated factors. In order to identify crucial factors for the aesthetic of web pages, we need to investigate the emotional semantics of aesthetic feelings, and the user's attitude toward aesthetic impression. Therefore, the goal of this study is to identify the crucial factors of the user's aesthetic impression in web-based learning systems, and to investigate the different emotional responses elicited by different cognitive style groups.

\section{Method}


We conducted four consecutive studies: an exploratory survey of semantic differences in web pages (stimuli), a study of emotional semantics (adjectives), an experimental survey of stimuli and adjective pairs, and Cognitive Styles Analysis (CSA).

\section{Survey on Stimuli}

We investigated users' perception and preference which affects how the web pages of a webbased learning system are selected. 105 undergraduate students (50 males and 55 females) were asked to write down approximately 10 web-based learning systems for image editing software which they visited or used frequently. The participants were also encouraged to explain the reasons for their selections. All of the subjects were aged from 18 to 25 , which corresponded to the main age groups that participated in web-based learning systems.

Then, nine web-based learning systems (Table 1) were selected for the following studies on the basis of those put forward.

Table 1. The nine web-based learning systems

PIXEL2LIFE (2010, March 24) http://www.pixel2life.com/tut orials/adobe_photoshop/ Heytalk (2010, March 24) http://www.facebook.com/heytalk 68 PS (2010, March 24) http://www.68ps.com/

Spoono (2010, March 24) http://www.spoono.com/photoshop/ Intelligent Group (2010, March 24) http://www.twisu.com.tw/ Muse craft (2010, March 24) http://musecraft.myweb.hinet.net/photoshop.html Psdtuts+ (2010, March 24) http://psd.tutsplus.com/ Unlimited design (2010, March 24) http://www.wuxiansj.com/article/pm/photoshop/ Flycan (2010, March 24) http://www.flycan.com.tw/ 
In this survey, stimuli subjects were limited to image editing software, such as Adobe Photoshop, and only the main web page was used as the stimulus in each case. Note that this experiment focuses on first impressions and allows the user to absorb the layout of static visual stimuli on a web page, hence acoustic and animated stimuli are ignored. Therefore, the nine most frequently visited websites were selected from 44 primary websites proposed. All the web layouts were captured on 24 March and surveyed between 5 and 25 May 2010.

\section{Survey on Semantic Adjectives}

In the second survey, the 105 students were asked to write down 10 emotional adjectives which define a good web-based learning system, such as beautiful, comfortable etc. Moreover, in order to have high reliability and a natural response, some adjectives were adapted to Mehrabian and Russell's (1974) measures of pleasantness and arousal in this survey. The total number of adjectives collected from students was 110 in the preliminary phase. Then five professional web designers were asked to screen for 15 suitable adjective pairs for expressing the measurement of web-based learning systems.

The 15 opposite adjective pairs are as follows: exciting-unexciting, interesting-boring, creative-traditional, attractive-unattractive, satisfied-unsatisfied, refined-rough, variousuniform, varied- monotonous, aroused-unaroused, professional-amateur, clear-confused, readable-unreadable, organized-chaotic, simple-complex, and regular-irregular. All variables have high reliability coefficients and Cronbach $\alpha$ was 0.87 , which is larger than 0.7 within goodness of fit, derived from a reliability analysis.

\section{Experimental Procedure}


There were 122 participants, consisting of 69 female and 53 male undergraduate students (different from the previous two surveys). The participants' ages ranged from 18 to 25, with an average age of 21.3. All participants were required to be familiar with basic computer functions and have never used experimental stimuli before this experiment. They participated voluntarily in the study.

In order to clearly investigate user's perceptions, we developed a system for an online survey. The nine web pages were used as stimuli in the survey. On each page of the survey, one of the nine web pages that were employed in this experiment was shown on the left, and the 15 aesthetic adjective pairs were shown on the right, with seven-point Likert scales. All participants were asked to look at each web page from one to nine as they appeared in random order on computer screens. Users could view the web page and at the same time indicate how much they felt the responses described by each of the 15 opposite adjective pairs. The system did not allow the users to go back to the previous pages and change their initial opinions. Each stimulus was randomly assigned to view only a single web page and participants were asked to view each web page for same amount of time (30 seconds). Those stimuli were not able to click on the links on the web page.

\section{Cognitive Styles Analysis}

Cognitive Styles Analysis (CSA) (Riding, 1991) be the most appropriate measure in personal cognitive behavior of educational research. The results of Cognitive Styles Analysis break down as follows:

1) The software outputs ratio, which can be converted into categories of wholist, intermediate and analytic (Riding, 1998); 
2) The actual sample consisted of 39 volunteers from the last stage of the online survey: 14 women and 25 men. Those who went on to volunteer for the CSA test included 6 analytic, 9 intermediate, and 24 wholist students.

\section{Analysis Method}

Factor analysis of the collected data was performed to separate several main factors for adjective pairs. In addition, MDS was conducted to convert the resulting similar data into a perceptual space representation (perceptual map) to further describe each group's features. The MDS procedure used in this study was SPSS ALSCAL. A perceptual map gives us a direct and obvious view of the relative position and degree of intimacy (the closer in distance, the more intimate in relationship) but it is not accurate enough to capture the classification detail of web-based learning systems. We need a result cluster to carry out classification and explanation. Therefore, five expert designers were invited to identify the number of links, number of graphics, and the amount of text as web page visual characteristics for further analysis.

\section{Results and Discussion}

Various analyses were conducted of the data collected from the above surveys to derive results for each survey in this study. These results will be described and discussed individually in the following sections.

\section{Main Visual Factors}

The data collected from the SD survey were processed by factor analysis. The result, as shown in Table 2, reveals that the 15 opposite adjective pairs were used to formulate a first impression of the web-based learning systems. They can be classified into two main factors 
with 60.386 of variance explained. A principal component analysis was used on classification. The analysis result showed two main factors with eigen values larger than 1.0, with explained variances being $43.74 \%$ and $16.65 \%$, respectively. KMO is 0.916 within high acceptable limits. The factor loadings of the two factors for the variables are plotted against each other.

The first cluster, which accounts for $43.74 \%$ of the variance, includes the adjective pairs: exciting - unexciting, interesting - boring, creative - traditional, attractive - unattractive, satisfied - unsatisfied, refined - rough, various - uniform, varied - monotonous, aroused - un aroused, and professional - amateur. This factor may be interpreted as the factor of visual complexity and richness. The second cluster, which accounts for $16.65 \%$ of the variance and includes the adjective pairs: clear - confused, readable - unreadable, organized - chaotic, simple - complex, and regular - irregular, may be interpreted as the factor of order and logical organization. See Figure 1.

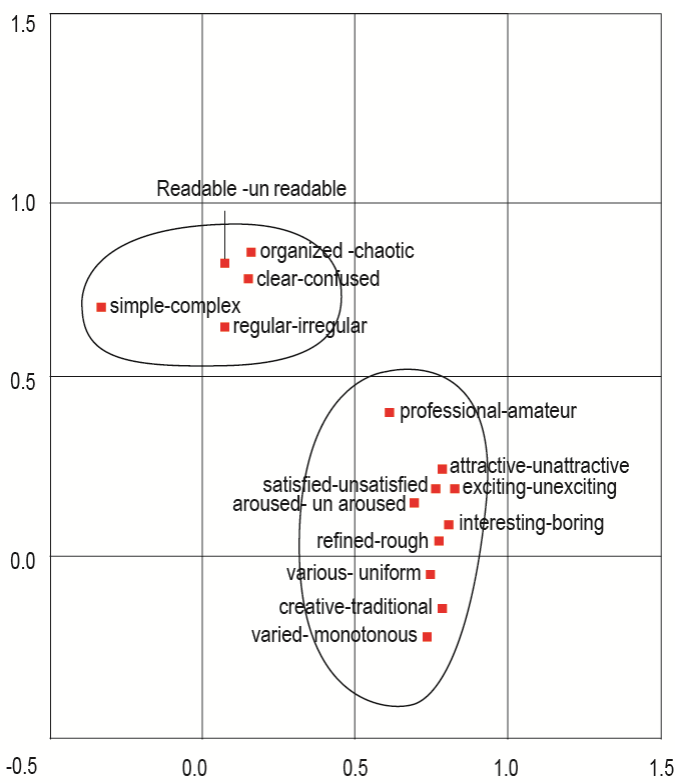

Figure 1. The Locations of 15 Adjective Pairs in the Space Derived From Factor Analysis 


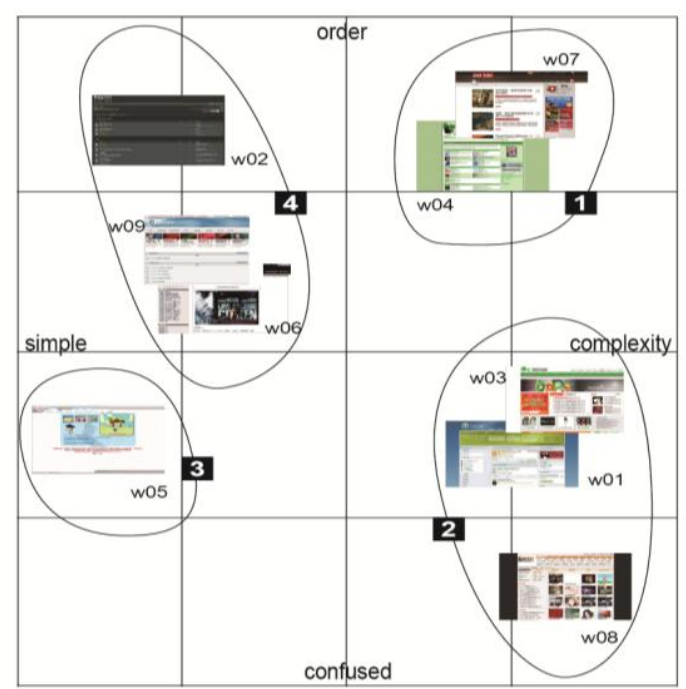

Figure 2. The four main groups were clustered in the perceptual map. 
Compared with the cognitive style grouping, intermediate students showed a higher average amount of opposite adjective pairs than wholist and analytic students. The statistics results showed the differences in the mean for students in the three cognitive style groups to be significant $(\mathrm{p}<0.05)$ in the order factor of 15 opposite adjective pairs. Analysis of variance (ANOVA) revealed that there were cognitive style group differences. The result of the Tukey test showed a significant effect of the complexity factor, satisfied-unsatisfied $(F(2)=3.600$, $\mathrm{p}<0.05)$. On the other hand, The result of the Tukey test showed a significant effect of the order factor, organized -chaotic $(\mathrm{F}(2)=3.040, \mathrm{p}<0.05)$, clear-confused $(\mathrm{F}(2)=7.819, \mathrm{p}<0.01)$, and readable -unreadable $(\mathrm{F}(2)=7.732, \mathrm{p}<0.01)$. These data (Table 2) indicate that intermediate students show higher emotional feeling (response) than wholist and analytic students towards the web-based learning system. See Table 2.

Table 2. The factor loading of 15 aadjective pairs were cluster to complexity and order factors and post hoc analysis

\begin{tabular}{|c|c|c|c|c|c|c|}
\hline \multirow[t]{2}{*}{ Factors } & \multirow[t]{2}{*}{ Adjective Pairs } & Factor & Loading & $\mathrm{F}$ & $\mathrm{P}$ & Post hoc \\
\hline & & \multicolumn{5}{|l|}{ Value } \\
\hline Complexit & exciting-unexciting & 0.83 & 0.18 & 1.783 & 0.169 & \multirow{10}{*}{$\mathrm{I}>\mathrm{A}$} \\
\hline \multirow[t]{9}{*}{$\mathrm{y}$} & interesting-boring & 0.81 & 0.08 & 0.143 & 0.866 & \\
\hline & creative-traditional & 0.78 & -0.14 & 0.206 & 0.814 & \\
\hline & attractive-unattractive & 0.78 & 0.22 & 0.223 & 0.800 & \\
\hline & satisfied-unsatisfied & 0.77 & 0.18 & 3.600 & $0.028 *$ & \\
\hline & refined-rough & 0.77 & 0.04 & 0.246 & 0.782 & \\
\hline & various- uniform & 0.75 & -0.05 & 1.410 & 0.245 & \\
\hline & varied- monotonous & 0.74 & -0.22 & 1.045 & 0.352 & \\
\hline & aroused- unaroused & 0.69 & 0.16 & 1.142 & 0.320 & \\
\hline & professional-amateur & 0.61 & 0.39 & 1.920 & 0.147 & \\
\hline \multirow[t]{5}{*}{ Order } & clear-confused & 0.16 & 0.82 & 7.819 & $0.000^{*}$ & $\mathrm{I}>\mathrm{A}$ \\
\hline & & & & & $*$ & $\mathrm{I}>\mathrm{W}$ \\
\hline & \multirow[t]{2}{*}{ readable -unreadable } & \multirow[t]{2}{*}{0.08} & \multirow[t]{2}{*}{0.79} & \multirow[t]{2}{*}{7.732} & $0.000^{*}$ & $\mathrm{I}>\mathrm{A}$ \\
\hline & & & & & $*$ & $\mathrm{I}>\mathrm{W}$ \\
\hline & organized -chaotic & 0.16 & 0.77 & 3.040 & $0.048^{*}$ & $\mathrm{I}>\mathrm{A}$ \\
\hline
\end{tabular}




\begin{tabular}{lllll}
\hline simple-complex & -0.34 & 0.67 & 2.417 & 0.090 \\
regular-irregular & 0.08 & 0.64 & 1.204 & 0.301 \\
\hline Eigenvalue & 10.65 & 4.05 & & \\
Percentage & 43.74 & 16.65 & & \\
Cumulative & 43.74 & 60.39 & & \\
Percentage & & & & \\
\hline
\end{tabular}

$\mathrm{W}:$ wholist. I: intermediate. A: analytic; $* \mathrm{p}<0.05 ; * * \mathrm{p}<0.01$

\section{Multidimensional Scaling}

The results of MDS, RSQ and Stress were indexed to access the reliability and validity of the perceptual map. To RSQ, the larger, the better with acceptance beyond 0.60; while to Stress, the smaller, the better with acceptance within 0.10 . The results indicate two-dimensional solutions with the RSQ value and the corresponding Stress measure being 0.977 and 0.065. The result was a fairly acceptable perceptual space of two dimensions through an appropriate rotation, as shown in Figure 2. From this perceptual map, we find that the web page visual layout located on the upper side shows more order/logical organization than that on ther side; while the web page visual layout located on the right side is more complex/rich than that on the left.

This observation is further verified by the high correlation between the $\mathrm{X}$ coordinate of the perceptual map and its corresponding complexity vs. simple scores; and between the Y coordinate of the perceptual map and its corresponding order vs. confused scores in the previous SD survey.

The perceptual map (Figure 2) classified the nine websites into four types. The first type shown in two web pages: w07 and w04. The second type includes three web pages: w01, w03, and w08. The third type is see above comment only one web page: w05. The last type See above three web pages: w02, w06, and w09. Five expert designers were invited to define each web page's fundamental characteristics and refer to statistical data (see Table 3), until they unanimously agreed to identify the following four distinct groups:

1) Professional image type: The main features consist of richness elements, including text, graphics, neutral color tone, structural layout and appropriate spaces on the web page. A 
pleasing color scheme and clear teaching cases are shown on those web pages, and they obtain high scores in complexity and order factors (see Table 3). The result indicates that complexity and order are both important visual factors in the first impression gained when browsing a website.

2) Largely graphics type: Multiple graphics, vivid color but minimum text. The layout is arranged predominantly symmetrically and horizontally. Some web pages of this type provide a forum platform function. Therefore, high amounts of link, text, and picture are found in this type (see Table 3). For instance, 46 links, 343 texts and 12 pictures are found in web page w03, more than in any of the other web pages.

3) Theme type: A large main graphic positioned in the center of the web page; mostly with less text. Web page w05 is a major teaching cases website, but there is only one picture on the home page. It seems that it doesn't easily attract users' interest.

4) Mainly informative type: Multiple text blocks and sections provide abundant information, but there are fewer graphics and a mono color tone. This type of website is seen in forums and blogs. There are more text lists or text contents than in other types of web layout, and only few colors and icons. For instance, web page w02 is a standard discussion forum website on Photoshop skills, and features a dark background, with white text and tables.

The complexity dimension refers to the degree to which a web page contains graphics, text and links. For example, w03 and w08 web pages refer to high complexity. And the order dimension signifies the degree to which the web page is clear and easy to read. For example, w07, w04 and w02 web page layouts exhibit a high degree of order. 
Table 3. Every item's statistical data were shown in the nine web pages

\begin{tabular}{lllllllll}
\hline \multirow{2}{*}{ No. } & \multirow{2}{*}{ Website name } & Type* links & text & \multicolumn{3}{c}{ Pictur Complexi } & Crder & Mean \\
& & & & & es & ty & & \\
\hline w01 & PIXEL2LIFE & MI & 43 & 78 & 8 & 0.578 & 0.293 & 0.483 \\
\hline w02 & Heytalk & LG & 26 & 265 & 1 & 0.299 & 0.947 & 0.515 \\
\hline w03 & 68 PS & MI & 46 & 343 & 12 & 0.784 & 0.598 & 0.722 \\
\hline w04 & Spoono & PI & 38 & 193 & 16 & 0.861 & 0.933 & 0.885 \\
\hline w05 & $\begin{array}{l}\text { Intelligent } \\
\text { Group }\end{array}$ & TT & 11 & 34 & 6 & 0.032 & 0.907 & 0.324 \\
\hline w06 & Muse craft & LG & 44 & 203 & 2 & 0.292 & 0.690 & 0.425 \\
\hline w07 & Psdtuts+ & PI & 22 & 134 & 13 & 0.748 & 0.888 & 0.795 \\
\hline w08 & Unlimited & MI & 84 & 127 & 23 & 0.629 & 0.235 & 0.498 \\
\hline W09 & Flycan & LG & 22 & 104 & 8 & 0.179 & 0.662 & 0.340
\end{tabular}

* PI: professional image type, LG: largely graphics type, TT: theme type, MI: mainly informative type.

Note: numbers of links, text, and pictures refer to the total amount on each web page.

\section{Conclusion and Limitations}

The results of the study show that the user's first impression determines their interest in visiting the website. The impression given by a home page was closely related to users' initial emotional responses. A web-based learning system is like many different kinds of instructors on the Internet. Novice learners tend to like to choose a website which gives a good impression, shows more teaching cases, is easy to read and has a clear webpage layout. Park et al. (2004) think aesthetic impressions are not necessarily unidirectional. Secondary emotions can be regarded as major types of aesthetic impressions. Thus, particularly for younger users is possible for aesthetic impressions to include feelings or secondary perceptions for younger users, such as 'lovely,' 'hope,' 'cool,' or positive feelings which are not limited to the dimension of beauty alone. 
Visual complexity and visual order were dependent on individual perception and cognitive style. Individuals' perceptions in the differentiation of web-based learning systems express a perceptual map constituted by two dimensions of complexity vs. simple, and order vs. confused. The result is closely related to Arnheim (1966) and Deng and Pool (2010). Arnheim (1966) has argued that order and complexity cannot exist without each other: order is needed for individuals to deal with high complexity as "complexity without order produces confusion", and some degree of complexity is necessary to bring interest to high order "as order without complexity causes boredom". Therefore, visual complexity and order factors should coexist in web-based learning systems, because an opportune combination within visual complexity and visual order easily enhances professional or trusting feeling. Similar feelings are also dependent on a positive dimension, i.e., higher scores on positive adjectives resulted in a higher factor of satisfaction toward the web page.

To sum up, the findings from the analysis suggest that the intermediate students showed higher emotional responses than the wholist and analytic students who exhibited different emotional responses, especially in order factors, when viewing a webpage. The present findings indicate that wholist, intermediate and analytic students have unique ways of perceiving a webpage. These characteristics of perception reflect some aspects of Nisbett's (2003) proposition about cognition style. It is suggested that webpage designers should be aware of the cognitive differences existing in wholist, intermediate and analytical groups, and of their target user's specific cognitive style. They would thereby enhance the user's perception of web-based learning systems.

Due to the limitation of resources, the impressions investigated in this study did not include all kinds of websites. Instead, we focused on free web-based learning systems (no joining fees) from amongst the students' favorites. This may have somewhat influenced the web page factor evaluations. The interactions among some usability and web page contents may have influenced the user's perception and preference, however due to the complexity of those problems, they were not considered in this study. 


\section{References}

Arnheim R. (1966). Order and Complexity in Landscape Design. In R. Arnheim (ed.), Toward a Psychology of Art (pp. 123-125). Berkeley, CA: University of California Press.

Bucy E. P. (2000). Emotional and Evaluative Consequences of Inappropriate Leader Displays. Communication Research, 27(2), 194-226.

Chuanga M. C, \& Ma, YC. (2001). Expressing the expected product images in product design of micro-electronic products. International Journal of Industrial Ergonomics, 27, 233245.

Cockton G. (2002). From doing to being: bring emotion into interaction, Interacting with Computers, 14, 89-92.

Deng L., \& Poole M. S. (2010). Affect in web interfaces: a study of the Impacts of Web Page Visual Complexity and Order, MIS Quarterly, 34 (4), 711-730.

Dillon A. (2001). Beyond Usability: Process, Outcome and Affect in Human-Computer Interactions, Canadian Journal of Library and Information Science, 26(4), 57-69.

Green P. E., Carmone F.J., \& Smith, S.M. (1989). Multidimentional Scaling-Concepts and Applications. Boston: Allyn \& Bacon.

Jindo T., Hirasago K., \& Nagamachi M. (1995). Development of a design support system for office chairs using 3-D graphics, International Journal of Industrial Ergonomics, 15(1), 49-62.

Lavie T., \& Tractinsky N. (2004). Assessing dimensions of perceived visual aesthetics of web Sites. International Journal of Human-.omputer Studies, 60(3). 269-298.

Lee S., \& Nagamachi M. (1996). Kansei Human Engineering. Seoul: Yangyonggak.

Nisbett R. E. (2003). The geography of thought: How Asians and Westerners think differently - and why. New York: Free Press.

Norman, D. A. (2002). Emotion and Design: Attractive Things Work Better, Interactions Magazine, 9(4). 36-42.

Norman D. A. (2003). Emotional Design: Why We Love (Or Hate) Everyday Things. New York: Basic Books.

Osgood C. E., Suci G. J., \& Tannenbaum P.H. (1957). The Measurement of Meaning., Chicago: University of Illinois Press.

Park S. (2004). Critical factors for the aesthetic fidelity of web pages: empirical studies with professional web designers and users, Interacting with Computers, 16(2). 351-376. 
Riding R. J., \& Cheema I. (1991). Cognitive Styles: an Overview and Integration, Educational Psychology, 11, 193-215.

Riding R. J., \& Rayner G. (1998). Cognitive Styles and Learning Strategies. London: David Fulton Publishers.

Schenkman B. N., \& Jönsson F. U. (2000). Aesthetics and preferences of web pages, Behaviour \& Information Technology, 19(5). 367-377.

Thompson, W. F., \& Robitaille B. (1992). Can composers express emotions through music?, Empirical Studies of the Arts, 10(1), 79-89.

Tsai T. W., Chang, T. C., Chuang M. C., \& Wang, D. M. (2008). Exploration in emotion and visual information uncertainty of websites in culture relations, International Journal of Design, 2(2), 55-66. 


\section{Websites}

PIXEL2LIFE (2010, March 24) http://www.pixel2life.com/tut orials/adobe_photoshop/

Heytalk (2010, March 24) http://www.facebook.com/heytalk

68 PS (2010, March 24) http://www.68ps.com/

Spoono (2010, March 24) http://www.spoono.com/photoshop/

Intelligent Group (2010, March 24) http://www.twisu.com.tw/

Muse craft (2010, March 24) http://musecraft.myweb.hinet.net/photoshop.html

Psdtuts+ (2010, March 24) http://psd.tutsplus.com/

Unlimited design (2010, March 24) http://www.wuxiansj.com/article/pm/photoshop/

Flycan (2010, March 24) http://www.flycan.com.tw/ 